\title{
Concurrent multiple intracranial and intramedullary tuberculoma: a rare form of neurotuberculosis
}

\author{
Vikram Singh Tanwar*, Harpreet Singh, Nikhil Govil, Sameer Arora, Ruchi Jagota \\ Department of Medicine, PGIMS Rohtak (124001), India
}

\author{
DOI Name \\ http://dx.doi.org/10.3126/jaim.v4i1.14177

\section{Keywords} \\ tuberculosis; central nervous system; tuberculoma

\section{Citation} \\ Vikram Singh Tanwar, Harpreet Singh, Nikhil Govil, \\ Sameer Arora, Ruchi Jagota. Concurrent multiple \\ intracranial and \\ intramedullary tuberculoma: a rare form of \\ neurotuberculosis. Journal of Advances in Internal \\ Medicine 2015;04(01):25-27.
}

\begin{abstract}
Central nervous system tuberculosis is a serious form of tuberculosis. Tuberculous CNS involvement can occur in the form of TB meningitis, tuberculous Vasculitis, tuberculoma and rarely brain abscess. Tubercular granulomas generally solitary and occur in the brain but they may be multiple and involve other areas such as spinal cord, epidural space and subdural space also. Tuberculoma in the spinal cord is rare. Concurrent occurrence of brain tuberculomas along with intramedullary spinal tuberculoma is even rarer. Only few cases have been reported in world literature. We are reporting a 28 years old female who presented with headache and progressive paraparesis in which imaging revealed intracranial and intramedullary tuberculoma and recovered completely with antitubercular therapy without any surgical intervention.
\end{abstract}

\section{INTRODUCTION}

Tuberculosis (TB) is one of the oldest diseases known to affect humans and is the major cause of death worldwide. India has the highest burden of tuberculosis in the world and one fourth of the global incident Tb cases occur in India annually. Central nervous system (CNS) involvement, one of the most devastating clinical manifestations of TB is noted in $5-10 \%$ of extrapulmonary TB cases, and accounts for approximately $1 \%$ of all TB cases. Tuberculoma, an uncommon manifestation of CNS TB, presents as one or more space occupying lesion and usually involves brain parenchyma ${ }^{1}$. Tuberculoma in the spinal cord is rare. Concurrent presence of tuberculomas in brain and spinal cord is even rarer. Only few cases have been reported in world literature.

\section{CASE REPORT}

Patient 28 years old female presented with 2 months history of headache, vomiting, vertigo triggered by abrupt head movements. Patient had history of weakness in bilateral lower limbs for 1 month. At the time of admission patient was unable to stand on her feet without support. There was no history of fever/ cough/ chest pain/ back pain/ band like sensation/ head trauma/ trauma to back / diplopia/ blurring of vision/ seizure like body movements/ loss of consciousness/ facial deviation/ dysphagia/ bladder bowel involvement. There was no past history suggestive of tuberculosis/ hypertension/ stroke. Patient was non-vegetarian by diet. Examination at this time revealed motor weakness of $3 / 5$ in both lower limbs with exaggerated deep tendon reflexes. There was no sensory abnormality found on examination. $X$ ray whole

\footnotetext{
* Corresponding author

Vikram Singh Tanwar

Department of Medicine, Pt. B.D.S. PGIMS,

Rohtak (124001) India

Email:drvikrampgi@gmail.com
} 
spine was almost within normal limit. NCCT head was done to find out the cause of chronic headache but it was almost normal except few small hypodensities seen in right frontal lobe region. Routine investigations like complete hemogram, liver function test and renal function test, viral serology was negative. MRI Brain and Spinal cord was planned that revealed multiple rounded lesions with surrounding edema in the bilateral cerebral hemisphere, cerebellum, brain stem, cervicomedullary junction and conus medullaris (Figure 1).

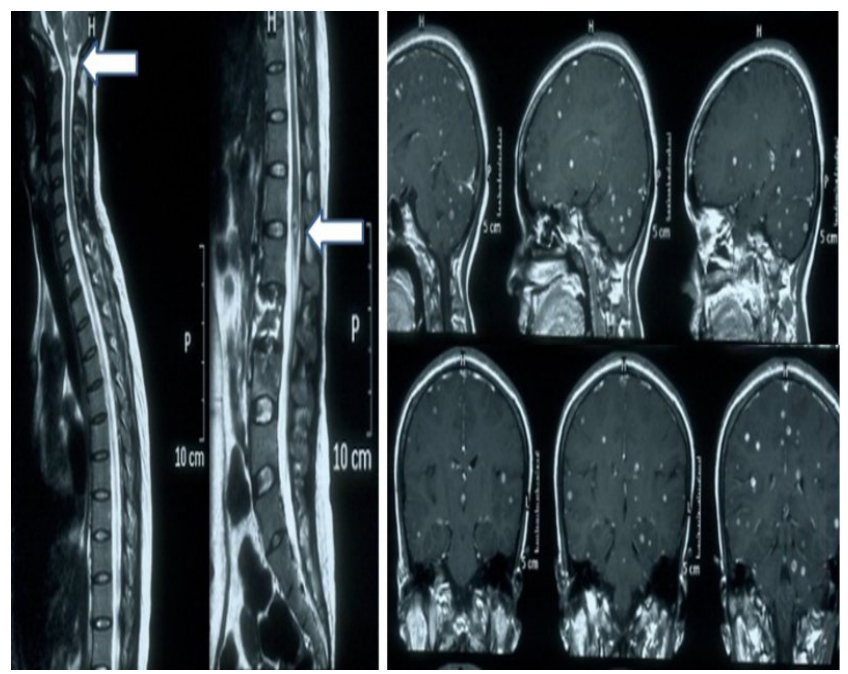

Figure 1: MRI brain and spinal cord on admission revealed multiple rounded lesions with surrounding edema in the bilateral cerebral hemisphere, cerebellum, brain stem, cervicomedullary junction and conus medullaris (see arrow head)

Biochemical and cytological examinations of CSF were normal. Mantoux was negative. Serology for neurocysticercosis was also negative. CECT chest revealed no pulmonary abnormality. So the final diagnosis made was caries spine with multiple tuberculomas in brain and spinal cord and patient was put on full dose ATT. In the first two months patient was administered Isoniazid $300 \mathrm{mg}$ daily, Rifampicin $600 \mathrm{mg}$ daily, pyrazinamide $1.5 \mathrm{gm}$ daily and Ethambutol $800 \mathrm{mg}$ daily. For the next ten months, Isoniazid (300mg) and rifampicin(600mg) once daily was planned. At the start of therapy, Dexamethasone $(24 \mathrm{mg} /$ day in three divided doses) was added to the regime for brain and spinal cord perilesional edema for 12 days. With treatment patient improved symptomatically. Power in the lower limbs improved from grade $3 / 5$ to $4 / 5$ within two weeks. After sixth months of ATT, power in the lower limbs improved to 5/5 and she became completely asymptomatic. Follow up MRI spinal cord showed complete disappearance of lesions in spinal cord and follow up MRI brain revealed marked reduction in number as well as size of tuberculomas with no perilesional edema (Figure 2). Same treatment was continued further to complete the 12 months course of ATT.

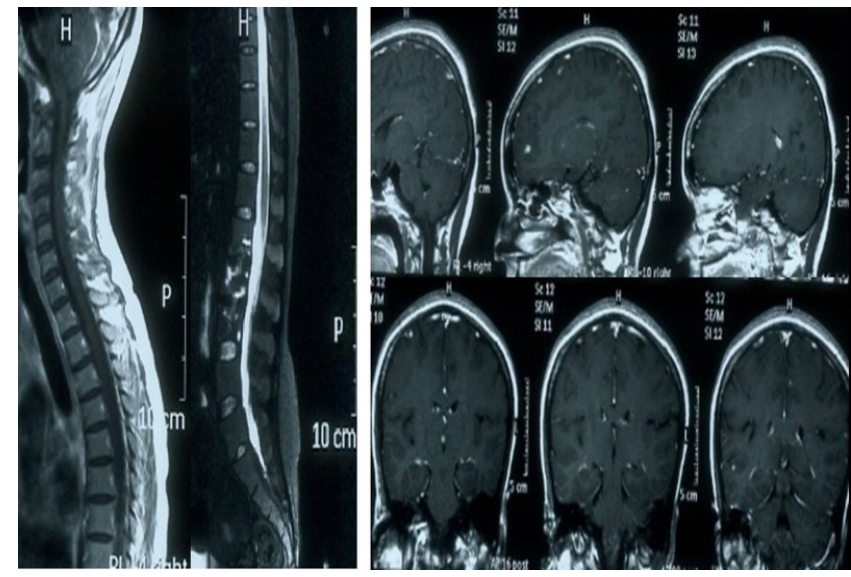

Figure 2: MRI brain and spinal cord after six months of treatment. MRI spinal cord showing disappearance of ring lesions and MRI brain showing marked reduction in number as well as size of tuberculomas with no perilesional edema..

\section{DISCUSSION}

Tuberculosis can involve almost any organ of the body. CNS tuberculosis is a serious form of extra-pulmonary tuberculosis and is associated with significant mortality and morbidity. Risk factors for CNS tuberculosis are children ${ }^{2}$, HIV coinfection ${ }^{3}$, malnutrition, and recent measles in children, alcoholism, malignancies, and the use of immunosuppressive agents in adults. In the central nervous system (CNS) it can cause tubercular meningitis (TBM), tuberculoma, abscess, or other manifestations. TBM is the most frequent form of CNS tuberculosis and constitutes approximately $1 \%$ of all forms of tuberculosis ${ }^{1}$. Tuberculomas are firm, avascular, granulomatous masses of variable size ranging from few $\mathrm{mm}$ to $4 \mathrm{~cm}$. Lesions may occur in the brain, spinal cord, subarachnoid, subdural, or epidural space. Intracranial tuberculoma can occur at any age. In children they are usually infratentorial, but supratentorial lesions predominate in adults. Solitary tuberculomas are more common (almost three times) than multiple lesions. Clinical features of intracranial TB granulomas result from a combination of factors, such as lesion number, stage, localization and the patient's immune response status against granuloma ${ }^{4}$. Signs and symptoms in the clinical process of brain tuberculomas are generally silent and the complaints gradually increase, and usually presents with headache, seizures, focal neurologic deficits, and papilledema ${ }^{5}$.

Involvement of spinal cord compared to that of brain occurs in the ratio of 1:42. Even in TB endemic areas, intra-medullary tuberculoma is rarely seen. The incidence of intramedullary tuberculoma is two in 100,000 cases of all tuberculosis and constitutes only 0.2 to $5 \%$ of all CNS tuberculomas ${ }^{6}$. Concurrent occurrence of intracranial tuberculomas and intramedullary spinal tuberculoma is even rarer. Only few cases have been 
reported in world literature. Yen et $\mathrm{al}^{7}$ reported a case with known pulmonary tuberculosis that developed symptoms of spinal cord compression. Imaging revealed an intramedullary tuberculoma along with multiple intracranial tuberculomas. Thacker et $\mathrm{al}^{8}$ reported a young girl who presented with progressive paraparesis in whom imaging revealed intramedullary tuberculoma with incidentally discovered multiple intracranial tuberculoma. Our patient had multiple ring enhancing tuberculomas in brain as well as spinal cord. In the brain, granulomas were found in both infratentorial and supratentorial region of brain. In the spinal cord they were found in very uncommon areas such as cervicomedullary junction and conus medullaris due to which our patient developed paraparesis.

On CT imaging, tuberculomas are characterised as low- or highdensity and rounded lesions with an irregular wall of varying thickness and show intense homogenous or ring enhancement after contrast administration ${ }^{9}$. MRI is the imaging modality of choice for diagnosis of these lesions and avoids the need for invasive procedure. The MRI features of tuberculoma depend on whether the lesion is non-caseating with a solid centre, or caseating with a liquid centre. The noncaseating granulomas are hypointense on T1-weighted images and hyperintense on T2-weighted images; after contrast administration the lesion usually shows homogenous enhancement. The second type of tuberculoma is hypointense or isointense on both T1 and T2-weighted images; after contrast administration there is ring enhancement ${ }^{10}$. These types of granuloma have variable degree of perilesional edema.

The differential diagnosis of tuberculomas (intracranial as well as intramedullary) includes cysticercal granulomas, pyogenic bacterial abscess, toxoplasmosis and neoplastic lesions such as astrocytoma, metastasis or lymphoma.

Medical management is recommended mode of treatment for these tuberculomas and has yielded good results in different series. In our patient, we advised our patient a full dose ATT course and the response was very good. Patient recovered markedly with disappearance of lesions in spinal cord and reduction in number as well as size of lesions in brain on follow up MRI imaging.

\section{CONCLUSION}

We report a unique case of multiple intracranial and intramedullary tuberculoma with paraparesis. We suggest that MRI of the spinal cord should be performed in the case of multiple intracranial tuberculomas because of the possible presence of early asymptomatic intramedullary tuberculomas.

\section{REFERENCES}

1. Raviglione MC, O'Brien RJ. Tuberculosis. In: Longo DL, Fauci AS, Kasper DL, Hauser SL, Jameson JL, Loscalzo J editors. Harrison's Principles Internal Medicine. 18th ed. New York: McGrawHill; p.1340-59.

2. Farer LS, Lowell AM, Meador MP. Extrapulmonary tuberculosis in the United States. Am J Epidemiol 1979;109: 205-217.

3. Dubé MP, Holtom PD, Larsen RA. Tuberculous meningitis in patients with and without human immunodeficiency virus infection. Am J Med 1992;93: 520-524.

4. Nicolls DJ, King M, Holland D, Bala J, del Rio C. Intracranial tuberculomas developing while on therapy for pulmonary tuberculosis. Lancet Infect Dis 2005;5: 795-801.

5. Garcia-Monco JC. Central nervous system tuberculosis. Neurol Clin 1999;17: 737-759.
6. Suzer T, Coskun E, Tahta K, Bayramoglu H, Duzcan E. Intramedullary spinal tuberculoma presenting as a conus tumour: a case report and review of the literature. Eur Spine J 1998;7:168-71.

7. Yen HL, Lee RJ, Lin JW, Chen HJ. Multiple tuberculomas in the brain and spinal cord: a case report. Spine 2003; 28:E499-502.

8. Thacker MM, Puri Al. Concurrent intra-medullary and intracranial tuberculomas. J Postgrad Med 2004; 50:107-9.

9. Wasay $M$, Kheleani BA, Moolani MK, et al. Brain CT and MRI findings in 100 consecutive patients with intracranial tuberculoma. J Neuroimaging 2003;13:240247.

10. Shen WC, Cheng TY, Lee SK, Ho YJ, Lee KR. Disseminated tuberculomas in spinal cord and brain demonstrated by MRI with Gadolinium-DTPA. Neuroradiology 1993; 35:213-5. 